\title{
Harnessing the Extracellular Bacterial Production of Nanoscale Cobalt Ferrite with Exploitable Magnetic Properties
}

\author{
Victoria S. Coker, ${ }^{+, *}$ Neil D. Telling, ${ }^{\dagger}$ Gerrit van der Laan, ${ }^{+, *}$ Richard A. D. Pattrick, ${ }^{\dagger}$ Carolyn I. Pearce, ${ }^{\dagger}$ \\ Elke Arenholz, ${ }^{\S}$ Floriana Tuna," Richard E. P. Winpenny," and Jonathan R. Lloyd ${ }^{\dagger}$ \\ ${ }^{\dagger}$ School of Earth, Atmospheric and Environmental Sciences and Williamson Research Centre for Molecular Environmental Science, University of Manchester, Manchester, \\ M13 9PL, U.K., 'Diamond Light Source, Didcot, Oxfordshire, 0X11 0DE, U.K., ${ }^{\S}$ Advanced Light Source, Lawrence Berkeley National Laboratory, Berkeley, California 94720 , and \\ ISchool of Chemistry, University of Manchester, Manchester, M13 9PL, U.K.
}

ABSTRACT Nanoscale ferrimagnetic particles have a diverse range of uses from directed cancer therapy and drug delivery systems to magnetic recording media and transducers. Such applications require the production of monodisperse nanoparticles with well-controlled size, composition, and magnetic properties. To fabricate these materials purely using synthetic methods is costly in both environmental and economical terms. However, metalreducing microorganisms offer an untapped resource to produce these materials. Here, the Fe(III)-reducing bacterium Geobacter sulfurreducens is used to synthesize magnetic iron oxide nanoparticles. A combination of electron microscopy, soft X-ray spectroscopy, and magnetometry techniques was employed to show that this method of biosynthesis results in high yields of crystalline nanoparticles with a narrow size distribution and magnetic properties equal to the best chemically synthesized materials. In particular, it is demonstrated here that cobalt ferrite $\left(\mathrm{COFe}_{2} \mathrm{O}_{4}\right)$ nanoparticles with low temperature coercivity approaching $8 \mathrm{kOe}$ and an effective anisotropy constant of $\sim 10^{6} \mathrm{erg} \mathrm{cm}^{-3}$ can be manufactured through this biotechnological route. The dramatic enhancement in the magnetic properties of the nanoparticles by the introduction of high quantities of $\mathrm{C}_{0}$ into the spinel structure represents a significant advance over previous biomineralization studies in this area using magnetotactic bacteria. The successful production of nanoparticulate ferrites achieved in this study at high yields could open up the way for the scaled-up industrial manufacture of nanoparticles using environmentally benign methodologies.

KEYWORDS: cobalt ferrite $\cdot$ Geobacter sulfurreducens $\cdot \mathrm{Fe}(\mathrm{III})$-reducing bacteria $\cdot$ nanoparticles $\cdot$ magnetism

*Address correspondence to vicky.coker@manchester.ac.uk.

Received for review March 24, 2009 and accepted June 02, 2009.

Published online June 9, 2009. 10.1021/nn900293d CCC: \$40.75

๑) 2009 American Chemical Society

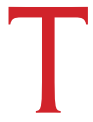
he production of ferromagnetic nanoparticles for pioneering cancer therapy, drug delivery, chemical sensors, catalytic activity, photoconductive materials, as well as more traditional uses in data storage embodies a large area of inorganic synthesis research. ${ }^{1-4}$ In particular, the addition of transition metals other than Fe into the structure of magnetite $\left(\mathrm{Fe}_{3} \mathrm{O}_{4}\right)$ has been shown to greatly enhance the magnetic properties of the particles, tailoring them to different commercial uses. ${ }^{1,2,5}$ However, synthesis of magnetic nanoparticles is often carried out at high temperatures with toxic solvents resulting in high environmental and energy costs. Additionally, these ferrite nanoparticles are not in- trinsically biocompatible, and to make them suitable for insertion into the human body is a rather intricate task.

A relatively unexplored resource for magnetic nanomaterial production is subsurface Fe(III)-reducing bacteria, as these microorganisms are capable of producing large quantities of nanoscale magnetite $\left(\mathrm{Fe}_{3} \mathrm{O}_{4}\right)$ at ambient temperatures. Metalreducing bacteria live in environments deficient in oxygen and conserve energy for growth through the oxidation of hydrogen or organic electron donors, coupled to the reduction of oxidized metals such as $\mathrm{Fe}(\mathrm{III})$ bearing minerals. ${ }^{6}$ This can result in the formation of magnetite via the extracellular reduction of amorphous Fe(III)-oxyhydroxides causing the release of soluble Fe(II) and resulting in complete recrystallization of the amorphous mineral into a new phase. ${ }^{7,8}$ Some previous studies have reported altering the composition of biogenic magnetite produced by $\mathrm{Fe}$ (III)-reducing bacteria for industrial and environmental applications. ${ }^{9,10}$ However, research into the commercial exploitation of bacteria to form magnetic minerals has focused primarily on magnetotactic bacteria which form magnetosomal magnetite internally using very different pathways to those bacteria forming magnetite outside the cell. ${ }^{11-14}$ Magnetotactic bacteria live at the sediment-water interface and use internal nanomagnets to guide them to their preferred environmental niche using the Earth's magnetic field. Since magnetotactic bacteria generally grow optimally under carefully controlled microaerobic conditions, the culturing processes for these organisms are challenging and result in low yields of nanomagnetite. Despite these limitations, magnetotactic bacteria 


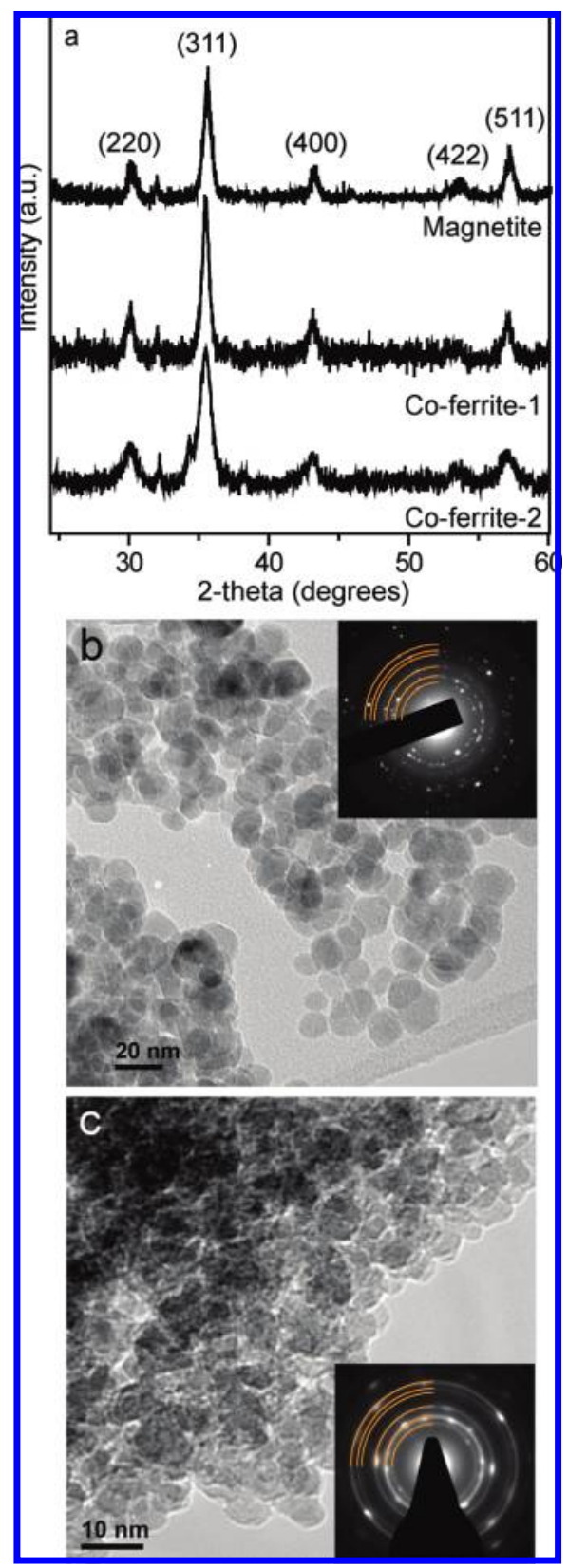

Figure 1. (a) X-ray diffraction of biogenic magnetite, Coferrite-1, and Co-ferrite-2. Transmission electron microscopy (TEM) with selected area diffraction (inset) for biogenic (b) magnetite and (c) Co-ferrite-2. Note the different scale bars of the TEM images.

have been shown to incorporate $\sim 1 \%$ Co into the magnetite structure in vivo, ${ }^{15}$ and $\mathrm{CoFe}_{2} \mathrm{O}_{4}$ was synthesized

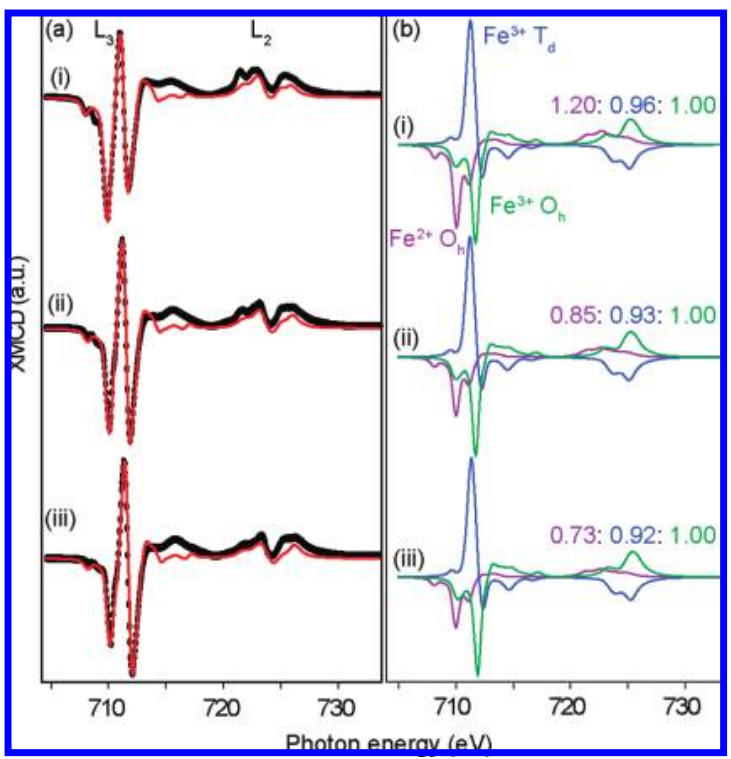

Figure 2. (a) Experimental Fe $L_{2,3}$ XMCD spectra (black circles) with best-fit spectra (red line) for biogenic (i) magnetite, (ii) Co-ferrite-1, and (iii) Co-ferrite-2. (b) For the best-fit spectrum of each biogenic sample, the decomposition into the spectra for the $\mathrm{Fe}^{2+} O_{h}, \mathrm{Fe}^{3+} T_{d}$, and $\mathrm{Fe}^{3+} O_{h}$ components is given. The color-coded numbers indicate the proportions of the components, and the error bar for each site occupancy is \pm 0.02 cations.

in vitro, ${ }^{14}$ altering the magnetic properties of the material formed. Although these previous studies are an important first step, in order to obtain the degree of control over the magnetic properties required by potential applications, Co must be incorporated into the spinel structure together with high nanoparticle yields. It is not clear at present how this could be achieved using the highly regulated intracellular magnetosome systems.

Here we present an alternative and efficient method to produce large quantities of highly crystalline magnetite and cobalt ferrite nanoparticles using the Fe(III)reducing bacterium, Geobacter sulfurreducens, at ambient temperatures through the extracellular dissimilatory reduction of Fe(III)-oxyhydroxides without and with addition of cobalt. A suite of three samples containing progressive additions of Co was produced for this study. A combination of transmission electron microscopy (TEM), X-ray diffraction (XRD), X-ray absorption (XA), and X-ray magnetic circular dichroism (XMCD) was used to determine the crystallographic structure,

TABLE 1. Structural and Magnetic Properties from Biogenic Magnetite and Co-Enriched Bioferrites

\begin{tabular}{|c|c|c|c|}
\hline sample & biomagnetite & Co-ferrite-1 & Co-ferrite-2 \\
\hline EDX Co/Fe ratio & $0.00: 1$ & $0.06: 1$ & $0.30: 1$ \\
\hline XRD crystallite size (nm) & 15 & 16 & 8 \\
\hline saturation magnetization, $M_{\mathrm{S}}\left(\mathrm{emu} \mathrm{g}^{-1}\right)$ at $298 \mathrm{~K}$ & 78 & - & 48 \\
\hline remanent magnetization, $M_{\mathrm{R}} / M_{\mathrm{S}}$ at $298 \mathrm{~K} /$ at $5 \mathrm{~K}$ & $0.06 / 0.25$ & - & $0.04 / 0.62$ \\
\hline coercivity, $H_{\mathrm{C}}(0 \mathrm{e})$ at $5 \mathrm{~K}$ & 360 & - & 7900 \\
\hline median particle diameter, $d_{0}(\mathrm{~nm})$ & - & - & 7.9 \\
\hline most probable diameter, $d_{p}(\mathrm{~nm})$ & - & - & 6.8 \\
\hline dispersion index, $\sigma_{d}$ & - & - & 0.38 \\
\hline
\end{tabular}




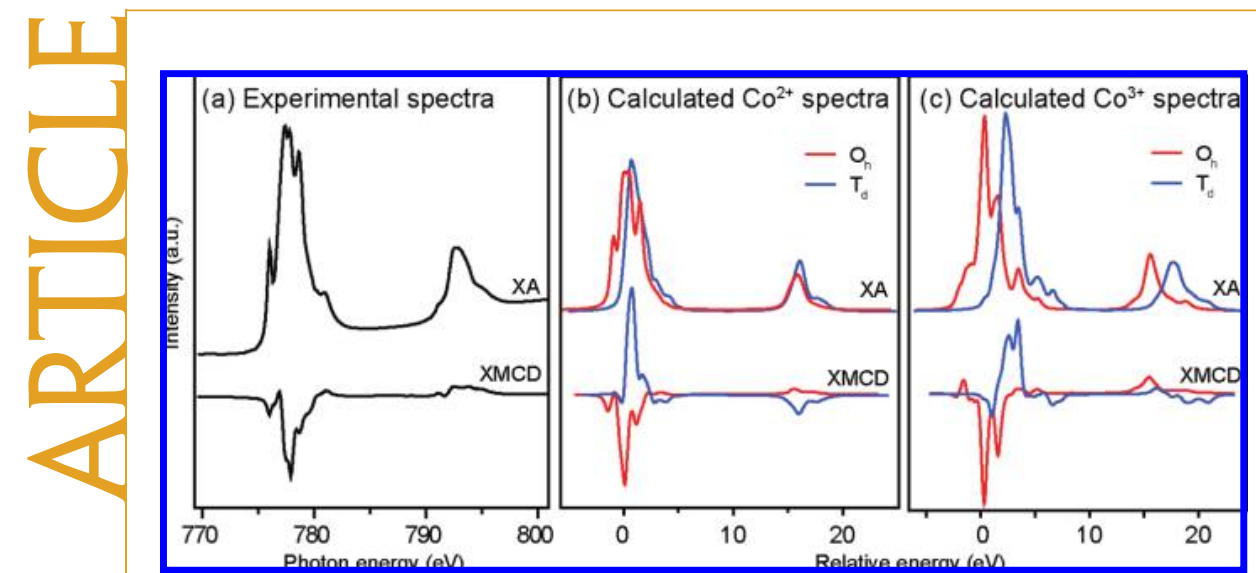

Figure 3. (a) Experimental Co $L_{2,3} X A$ and XMCD spectra for biogenic Co-ferrite-2. Calculated XA and XMCD spectra for (b) $\mathrm{Co}^{2+}$ and (c) $\mathrm{Co}^{3+}$ in octahedral (red line) tetrahedral (blue line) coordination. Calculations were done following the method of refs 18 and 19. values correlate well with the observed particle sizes in TEM images (Figure 1), confirming the single crystalline nature. In addition, the TEM images — as well as magnetization measurements discussed below-indicate a narrow size distribution of the particles, crucially important for many technological applications.

The cation distribution in the ferrite nanoparticles was investigated using X-ray magnetic circular dichroism (XMCD) at the Fe $L_{2,3}$ and $C o L_{2,3} X$-ray absorption edges. XMCD is the difference in the absorption of circularly polarized X-rays for opposite sample magnetization directions. Stoichiometric magnetite is an insite occupancies, and valence states of the Fe and Co cations within the spinel structure. Furthermore, superconducting quantum interference device (SQUID) magnetometry was used to determine the magnetic properties of these materials. The exceptional ability of the bacteria to manufacture a wide range of useful nanomaterials has until now been overlooked for production on a commercial scale.

\section{RESULTS AND DISCUSSION}

Biogenic magnetite and two related materials (biogenic Co-ferrite-1 and biogenic Co-ferrite-2) were produced from starting materials of $\mathrm{Fe}$ (III)-oxyhydroxide containing increasing amounts of co-precipitated $\mathrm{Co}$ (II), at ambient temperature and pressure using the metalreducing bacterium Geobacter sulfurreducens. Conversion of the amorphous Fe(III)-oxyhydroxide occurred during the reductive transformation by $G$. sulfurreducens to give a biomineral phase enriched with $\mathrm{Fe}(\mathrm{II})$, while control experiments without cells showed no change in acid-extractable Fe(II) (see Supporting Information). The yield of nanoparticles in this experiment was $1.6 \mathrm{~g}$ per $100 \mathrm{~mL}$ per day, which is comparable to or greater than yields given by other bacterial synthesis methods. ${ }^{14,16}$ Energy dispersive X-ray (EDX) analysis of the particles indicated that the Co content of the total cation distribution was 6 atom \% for Co-ferrite-1 and 23 atom \% for Co-ferrite-2. The latter is close to the Co content of stoichiometric Co-ferrite $\left(\mathrm{CoFe}_{2} \mathrm{O}_{4}\right)$. $\mathrm{X}$-ray diffraction (XRD) and selected area electron diffraction (SAED) in Figure 1 show that each of the samples gives only reflections arising from a ferrite structure. Interestingly, it was found that the particle size decreases for increasing Co content, as revealed by an overall broadening of the individual diffraction peaks in Figure 1a. Fitting the (311) diffraction peak with a Lorentzian line shape using the Scherrer equation ${ }^{17}$ gives the crystallite size for each material (Table 1). The XRD shows that the material is nanocrystalline, where the addition of 23 atom \% Co into the structure reduces the average crystallite size from $15-16$ to $8 \mathrm{~nm}$. These verse spinel, which contains tetrahedral $\left(T_{d}\right)$ and octahedral $\left[O_{h}\right]$ sites accommodating $\mathrm{Fe}^{2+}$ and $\mathrm{Fe}^{3+}$ cations according to the formula $\left(\mathrm{Fe}^{3+}\right)_{\mathrm{Td}}\left[\mathrm{Fe}^{2+} \mathrm{Fe}^{3+}\right]_{\mathrm{Oh}} \mathrm{O}_{4}$. Each specific cation in the spinel structure generates a unique XMCD signature that is determined by its valence state (number of $d$ electrons), site symmetry (i.e., $T_{d}$ or $O_{h}$ ), and magnetization (local moment) direction. The XA and XMCD spectra of the individual cations can be computed using atomic multiplet calculations. ${ }^{18,19}$ By fitting a weighted sum of these calculated spectra to the measured XMCD, the site distribution of the Fe cations can be obtained (for details see ref 20). Figure 2a shows the data together with the calculated fits for the Fe $L_{2,3}$ spectra of the biomagnetite and Co-enriched bioferrite samples. Figure $2 \mathrm{~b}$ gives the relative proportions of the three Fe components, $\mathrm{Fe}^{2+} \mathrm{O}_{h}, \mathrm{Fe}^{3+} T_{d}$, and $\mathrm{Fe}^{3+} \mathrm{O}_{h}$, which primarily make up the intensities of the three main peaks (negative, positive, and negative, respectively) in the Fe $L_{3}$ edge $(\sim 708-714 \mathrm{eV})$. A comparison of the biogenic materials shows that the most striking change with increasing Co amount is the gradual decrease in intensity of the leading negative peak in the Fe $L_{3}$ edge, corresponding to a decrease in $\mathrm{Fe}^{2+} \mathrm{O}_{h}$ content. The best-fit spectra give the values for the relative site occupancies as indicated in Figure $2 b$. The $\mathrm{Fe}^{2+} / \mathrm{Fe}^{3+}$ ratio in biogenic magnetite is $1.22: 2$, which is higher than the 1:2 ratio expected for stoichiometric magnetite, which is due to an increased amount of Fe $d^{6} O_{h}$ as a result of the anoxic environment in which the magnetite was formed. ${ }^{20,21}$ Upon the addition of $\mathrm{Co}$, the amount of $\mathrm{Fe}^{2+}$ decreases rapidly, with only a small accompanying change in the amount of $\mathrm{Fe}^{3+}$ cations per formula unit. This implies that $\mathrm{Co}$ is predominantly replacing $\mathrm{Fe}^{2+}$ cations in octahedral sites.

The site occupancy and oxidation state of the Co can be directly assessed by examining the Co $L_{2,3}$ XA and XMCD spectra (Figure 3 ). The close similarity between the spectra for Co-ferrite-2 and synthetically produced $\mathrm{CoFe}_{2} \mathrm{O}_{4}$ thin films ${ }^{22}$ signifies that the bacteria were able to suitably accommodate Co in the ferrite 

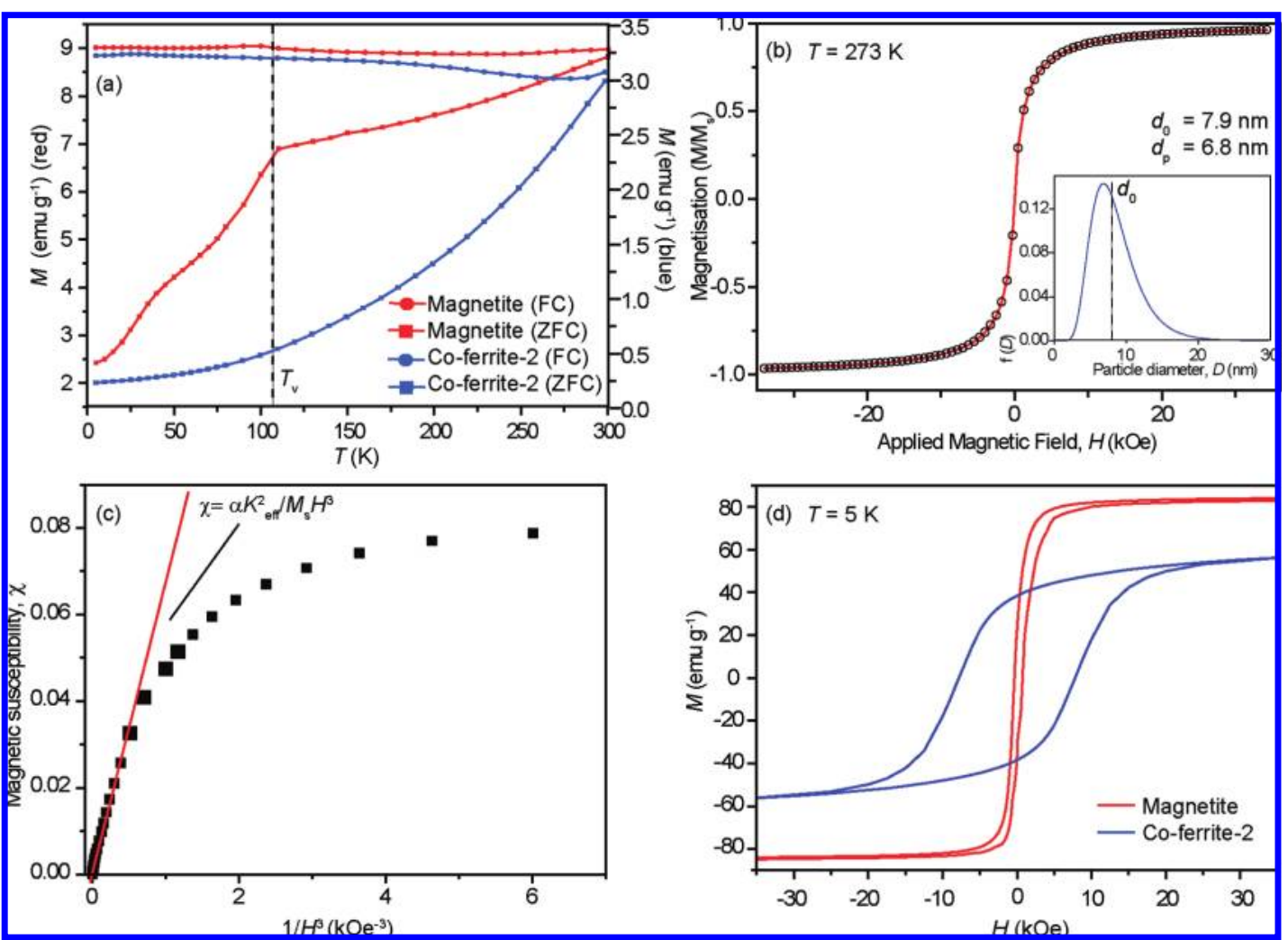

Figure 4. SQUID magnetometry measurements. (a) Zero-field cooled (ZFC) and field cooled (FC) curves for biogenic magnetite (red lines) and biogenic Co-ferrite-2 (blues lines). (b) Magnetic hysteresis curve at $273 \mathrm{~K}$ for Co-ferrite-2 with Langevin function fitting and (inset) derived particle size distribution. (c) Fit for biogenic magnetite using the Law of Approach to Saturation. (d) Magnetic hysteresis curves taken at $5 \mathrm{~K}$ for biogenic magnetite (red line) and Co-ferrite-2 (blue line).

structure. Calculated XA and XMCD spectra for $\mathrm{Co}^{2+}$ (Figure $3 \mathrm{~b}$ ) and $\mathrm{Co}^{3+}$ (Figure $3 \mathrm{c}$ ) in both $\mathrm{O}_{h}$ and $T_{d}$ coordination have been normalized and broadened to account for instrumental broadening for comparison with experimental spectra (Figure 3a). The strong resemblance of the experimental data to the calculated $\mathrm{Co}^{2+}$ $O_{h} X A$ and XMCD suggests that the Co cations reside primarily on these sites, particularly as the magnetic moments on the $T_{d}$ and $O_{h}$ sites are antiferromagnetically coupled, resulting in an XMCD with opposite sign for the two sites. The measurements show that the sign of the Co XMCD is the same as that of the Fe $O_{h} X M C D$, hence confirming the $O_{h}$ site occupancy of $\mathrm{Co}$. Further confirmation arises from the fact that the presence of the $\mathrm{Co}^{2+} \mathrm{O}_{h}$ coincides directly with the decrease in $\mathrm{Fe}^{2+}$ on octahedral sites found from the Fe $L_{2,3}$ XMCD. Nevertheless, a small amount $(\sim 10 \%)$ of Co residing in a different site or as $\mathrm{Co}^{3+}$ cannot be discounted as the fit is not perfect, particularly at the high energy side of the $L_{3}$.

The magnetic properties of the nanoparticles, graphically represented in Figure 4, are governed by the anisotropy energy, which constrains the nanoparticle magnetization to align along a specific direction known as the easy axis. The maximum anisotropy energy for a particular nanoparticle depends on the product of its volume, $V$, and anisotropy constant, $K$. Above the so-called blocking temperature, $T_{B}$, the thermal energy is sufficient to surmount the anisotropy energy

www.acsnano.org barrier and the nanoparticle magnetization is free to align in arbitrary directions. Thus, for an assembly of such nanoparticles, the net magnetization measured in the absence of an applied magnetic field (i.e., the remanent magnetization of the sample) will average out to zero and the particles are said to be in superparamagnetic state.

An estimate for the blocking temperature can typically be obtained from peaks in the field cooled (FC) and zero-field cooled (ZFC) magnetization curves. ${ }^{23}$ The experimental results for the biogenic magnetite and Co-ferrite-2 samples, obtained by SQUID magnetometry, are shown in Figure 4a. In this case, no peaks can be discerned in the ZFC/FC curves below $300 \mathrm{~K}$, suggesting that the blocking temperature is close to room temperature for both samples. The remanent magnetization for the Co-ferrite- 2 is lower than for biomagnetite (Table 1), and indeed, this sample displays the typical superparamagnetic magnetization curve at room temperature, as shown in Figure $4 \mathrm{~b}$.

The biogenic magnetite retains a small residual coercivity at room temperature (not shown), indicating an incomplete superparamagnetic state. The FC and ZFC curves in Figure $4 a$ for this sample contain a sharp feature at $\sim 110 \mathrm{~K}$ that is due to the Verwey transition $\left(T_{\mathrm{V}}\right){ }^{24}$ which is a well-known but poorly understood characteristic of pure $\mathrm{Fe}_{3} \mathrm{O}_{4}$ observed between 100 and $120 \mathrm{~K}$ depending on the exact composition of the ma- 
terial. ${ }^{25}$ This phase transition remains frequently unobserved in synthetic nanoparticulate magnetite, ${ }^{25}$ possibly due to lack of crystalline coherence compared to the bulk. The presence of a sharp transition temperature for the biogenic magnetite is a clear indication of the high quality crystal growth obtained by the biomineralization route, even for nanoparticles below $20 \mathrm{~nm}$. The absence of the transition for the Co-ferrite-2 sample, on the other hand, corroborates the finding that the Co entered the spinel structure. ${ }^{26}$

The exact shape of the magnetization curve for superparamagnetic particles depends on the magnetic particle volume and can be described by a Langevin function. ${ }^{25,27}$ For an assembly of polydisperse particles, the magnetic particle size distribution can be obtained from a fit to the magnetization curve taking a weighted sum over Langevin functions. ${ }^{28,29}$ Using the best-fit curve shown in Figure $4 \mathrm{~b}$ for the Co-ferrite-2 sample, the log-normal distribution is obtained (inset). It is seen that the median $(7.9 \mathrm{~nm})$ and most probable particle diameter $(6.8 \mathrm{~nm})$ of the log-normal distribution agrees well with the respective average crystallite size measured by XRD (Table 1) and particle size imaged in TEM (Figure 1). This again supports the notion that the particles are highly crystalline with little or no surface disorder. Furthermore, the dispersion index determined (Table 1) is comparable with chemically produced Coferrite particles of similar size. ${ }^{30}$

An estimation of the effective anisotropy constant, $K_{\text {eff, }}$ for the samples can be obtained by using the Law of Approach to Saturation. ${ }^{31,32}$ This allows us to deduce $K_{\text {eff }}$ from a fit to the magnetic susceptibility, $\chi$, versus $H^{-3}$ curve, where $H$ is the applied magnetic field, as is shown for the biomagnetite sample in Figure 4c. Measurements were carried out at low temperature $(5 \mathrm{~K}$, i.e., well below the blocking temperature) to ensure the samples are in the ferrimagnetic state. Assuming uniaxial anisotropy, the fits give $K_{\text {eff }}=2.4 \times 10^{5} \mathrm{erg} \mathrm{cm}^{-3}$ for biomagnetite and $K_{\text {eff }}=2.3 \times 10^{6} \mathrm{erg} \mathrm{cm}^{-3}$ for Coferrite- 2 . Thus, the Co inclusion in the spinel increases the magnetic anisotropy by an order of magnitude, which is consistent with the presence of $\mathrm{Co}^{2+}$ in octahedral sites found from the XMCD measurements and has also been observed for synthetic Co-ferrite particles. ${ }^{5,33}$ The results obtained for $K_{\text {eff }}$ are also comparable to the values of the first-order anisotropy constant for bulk magnetite $\left(\left|K_{1}\right| \cong 2.7 \times 10^{5} \mathrm{erg} \mathrm{cm}^{-3}\right.$ for the low temperature phase) ${ }^{31}$ and bulk Co-ferrite, $\mathrm{CoFe}_{2} \mathrm{O}_{4}\left(\left|K_{1}\right| \sim 2 \times 10^{6} \mathrm{erg} \mathrm{cm}^{-3}\right) .^{34}$

The coercivity, $H_{C}$, of the samples (i.e., the magnetic field needed for the magnetization to return to zero) can be related to the anisotropy constant according to $H_{\mathrm{C}}=0.958 \mathrm{~K} / \mathrm{MS}^{35}$ (assuming uniaxial anisotropy), where $M_{S}$ is the saturation magnetization (Table 1). At $5 \mathrm{~K}$, this gives a coercivity of $\sim 500$ and $\sim 7000$ Oe for biomagnetite and Co-ferrite-2, respectively. The measured values (shown in Figure $4 d$ and Table 1) are comparable to these estimated values derived from the measured anisotropy constants. However, it should be noted that the coercivity of 7000 Oe obtained above for the Coferrite-2 sample underestimates the experimental value of 7900 Oe. This is because, even at the highest measurement field of $7 \mathrm{~T}$, the sample was not completely saturated and therefore the Law of Approach to Saturation can provide only a lower limit to the effective anisotropy constant in this case. Further, we cannot rule out a contribution to the approach to magnetic saturation from noncollinear surface spins, ${ }^{36}$ which would tend make the surface of the particles magnetically hard compared to the inner core.

From the FC/ZFC curves shown in Figure 4a, it can be seen that the Co-ferrite-2 sample is only just superparamagnetic at room temperature (i.e., the blocking temperature is close to room temperature). A modest increase in either the particle diameter or the anisotropy constant (e.g., by slightly increasing the Co content) should be sufficient to produce nanoparticles with ferrimagnetic properties and large coercivities suitable for applications above room temperature. This is the subject of further work and will be presented elsewhere.

In summary, we have shown a dramatic enhancement in the magnetic properties of biogenically produced nanoparticles by introducing large quantities of Co into the spinel structure, which represents a major advance over previous biomineralization studies in this area. Inclusion of other transition metals into the spinel structure by Fe(III)-reducing bacteria to tailor the magnetic properties of nanoferrites could lead to a suite of materials required for different technological uses. The successful production of highly ordered crystalline nanoparticulate ferrites achieved here shows the potential for scaled-up industrial manufacture of nanoparticles using environmentally benign methodologies.

\section{METHODS}

Synthetic amorphous $\mathrm{Fe}$ (III)-oxyhydroxide was produced using the method of Lovley and Philips, ${ }^{37}$ where a $0.4 \mathrm{~mol}$ solution of $\mathrm{FeCl}_{3}$ is neutralized by $10 \mathrm{~N} \mathrm{NaOH}$ to $\mathrm{pH} 7$ and the solid then washed in deionized $\mathrm{H}_{2} \mathrm{O}$ until no $\mathrm{Cl}^{-}$ions remain. $\mathrm{CoCl}_{2} \cdot 6 \mathrm{H}_{2} \mathrm{O}$ was added prior to neutralization at a concentration of either 0.02 or $0.132 \mathrm{~mol}$ to create FeCo-oxyhydroxides which formed a suite of starting materials with an increasing amount of cobalt which result in samples Co-ferrite- 1 and Co- ferrite-2, respectively. The starting material was characterized using XRD to confirm that poorly crystalline material had formed. Geobacter sulfurreducens was obtained from our laboratory culture collection at the Williamson Centre for Molecular Environmental Science at the University of Manchester and grown under strictly anaerobic conditions at $30^{\circ} \mathrm{C}$ in modified fresh water medium. ${ }^{38}$ Sodium acetate $(20 \mathrm{mmol})$ and fumarate $(40 \mathrm{mmol})$ were provided as the electron donor and acceptor, respectively. All manipulations were done under an atmosphere of $\mathrm{N}_{2}-\mathrm{CO}_{2}$ 
(80:20). Late log-phase cultures of $G$. sulfurreducens were harvested by centrifugation at $4920 \mathrm{~g}$ for $20 \mathrm{~min}$ and washed twice in bicarbonate buffer $\left(\mathrm{NaHCO}_{3} 30 \mathrm{mmol}, \mathrm{pH}\right.$ 7.1) under $\mathrm{N}_{2}-\mathrm{CO}_{2}$ $(80: 20)$ gas prior to use. Aliquots of the washed cell suspension $(1.5 \mathrm{~mL})$ were added to sealed anaerobic bottles containing 28.5 $\mathrm{mL}$ of bicarbonate buffer to a final concentration of bacteria of $\sim 0.2 \mathrm{mg}$ protein per $\mathrm{mL}$. The following additions were made from anaerobic stocks as required: an $\mathrm{Fe}$ (III)-oxyhydroxide or $\mathrm{CoFe}$ (III)-oxyhydroxide suspension $\left(10 \mathrm{mmol} \mathrm{L}^{-1}\right)$, sodium acetate $(10 \mathrm{mmol})$, and anthraquinone 2,6 -disulfonate $(10 \mu \mathrm{mol})$. Bottles were incubated in the dark at $20^{\circ} \mathrm{C} ; 100 \mu \mathrm{L}$ of slurry was removed from each serum bottle periodically, using aseptic and anaerobic technique and analyzed for $0.5 \mathrm{~mol} \mathrm{HCl}-$ extractable $\mathrm{Fe}^{2+}$ employing the ferrozine method. ${ }^{37}$ After ferrite formation, samples were washed twice in deionized water to remove cells and buffer solution. Aliquots of the sample suspension were then dropped on carbon-coated copper grids and left to dry prior to introduction into the microscope.

Transmission electron microscopy (TEM) was carried out using a Phillips/FEl Tecnai electron microscope equipped with a field emission gun (FEG), EDX system (Oxford Instruments UTW ISIS), and Gatan imaging filter (GIF). All TEM images presented here are bright-field images obtained using an operating beam voltage of $300 \mathrm{keV}$. Selected area electron diffraction (SAED) patterns were acquired using an appropriate diffraction aperture. All (powder) X-ray diffraction (XRD) measurements were acquired over a $2 \theta$ range of $10-70^{\circ}$ with a step size of $0.02^{\circ}$ using a Bruker D8Advance with $\mathrm{Cu} \mathrm{K} \alpha_{1}$ source.

$\mathrm{X}$-ray absorption (XA) spectra at the $\mathrm{Fe}$ and $\mathrm{Co} L_{2,3}$ edges were obtained on beamline 4.0.2 at the Advanced Light Source (ALS), Berkeley, CA, using the eight-pole resistive magnet endstation. ${ }^{39}$ Samples were prepared by washing aliquots of the suspension in deionized water before drying under anaerobic conditions. The powders were then, in an anaerobic cabinet, mounted on carbon tape attached to the sample manipulator. XA was monitored in total-electron yield (TEY) mode, which has an effective probing depth of $\sim 4.5 \mathrm{~nm}$. At each photon energy of the spectrum, the XA was measured for two opposite magnetization directions by reversing the applied field of 0.6 T. After normalization to the incident beam intensity, the XMCD is obtained as the difference between the two XA spectra. ${ }^{40} \mathrm{To}$ obtain the cation distribution over the three Fe sites, the experimental XMCD was fitted by means of a nonlinear least-squares analysis, using the calculated spectra for each site. In these calculations, described in refs 18 and 19, the 10Dq crystal field parameters were taken as 1.2 and $-0.6 \mathrm{eV}$ for $\mathrm{Fe} O_{h}$ and $T_{d}$ sites and 0.9 and $-0.5 \mathrm{eV}$ for $\mathrm{Co}_{h}$ and $T_{d}$ sites. The results were convoluted by a Lorentzian of $\Gamma=0.3(0.5) \mathrm{eV}$ for the $L_{3}\left(L_{2}\right)$ edge to account for intrinsic core-hole lifetime broadening and by a Gaussian of $\sigma=0.2 \mathrm{eV}$ to account for instrumental broadening.

Magnetic measurements were performed on polycrystalline samples restrained in eicosane, using a Quantum Design MPMS-XL SQUID magnetometer equipped with a $7 \mathrm{~T}$ magnet. Zero-field cooled (ZFC) and field-cooled (FC) magnetization curves were recorded over $5-300 \mathrm{~K}$ temperature range with an applied magnetic field of 100 Oe. The diamagnetism of the sample holder and of eicosane was measured and extracted from the raw magnetic data.

Acknowledgment. This work was supported by the EPSRC grants EPSRC EP/D057310/1 and EP/D058767/1 and BBSRC grant BB/E003788/1. We would like to thank E. Mclnnes for assistance with SQUID measurements, G. Cliff for TEM support, and J. Waters for XRD support. The Advanced Light Source is supported by the Director, Office of Science, Office of Basic Energy Sciences, of the U.S. Department of Energy under Contract No. DE-AC02$05 \mathrm{CH} 11231$.

Supporting Information Available: Graph of Fe(II) concentration changes with time during the formation of biogenic ferrites. This material is available free of charge via the Internet at http://pubs.acs.org.

\section{REFERENCES AND NOTES}

1. Ritter, J. A.; Ebner, A. D.; Daniel, K. D.; Stewart, K. L. Application of High Gradient Magnetic Separation Principles to Magnetic Drug Targeting. J. Magn. Magn. Mater. 2004, 280, 184-201.

2. Kim, D.-H.; Nikles, D. E.; Johnson, D. T.; Brazel, C. S. Heat Generation of Aqueously Dispersed $\mathrm{CoFe}_{2} \mathrm{O}_{4}$ Nanoparticles as Heating Agents for Magnetically Activated Drug Delivery and Hyperthermia. J. Magn. Magn. Mater. 2008, 320, 2390-2396.

3. Safarik, I.; Safarikova, M. Magnetic Nanoparticles and Biosciences. Monatsh. Chem. 2002, 133, 737-759.

4. Jolivet, J.-P.; Tronc, E.; Chaneac, C. Synthesis of Iron OxideBased Magnetic Nanomaterials and Composites. C. $R$. Chim. 2002, 5, 659-664.

5. Sun, S.; Zeng, H.; Robinson, D. B.; Raoux, S.; Rice, P. M.; Wang, S. X.; Li, G. Monodisperse $\mathrm{MFe}_{2} \mathrm{O}_{4}(\mathrm{M}=\mathrm{Fe}, \mathrm{Co}, \mathrm{Mn})$ Nanoparticles. J. Am. Chem. Soc. 2004, 126, 273-279.

6. Caccavo, F., Jr.; Lonergan, D. J.; Lovley, D. R.; Davis, M.; Stolz, J. F.; Mclnerney, M. J. Geobacter sulfurreducens, sp. nov., a Hydrogen and Acetate-Oxidising Dissimilatory Metal Reducing Bacterium. Appl. Environ. Microbiol. 1994, 60, 3752-3759.

7. Coker, V. S.; Bell, A. M. T.; Pearce, C. I.; Pattrick, R. A. D.; van der Laan, G.; Lloyd, J. R. Time-Resolved Synchrotron Xray Powder Diffraction Study of Magnetite Formation by the Fe(III)-Reducing Bacterium Geobacter sulfurreducens. Am. Mineral. 2008, 93, 540-547.

8. Hansel, C. M.; Benner, S. G.; Neiss, J.; Dohnalkova, A.; Kukkadapu, R. K.; Fendorf, S. Secondary Mineralization Pathways Induced by Dissimilatory Iron Reduction of Ferrihydrite under Advective Flow. Geochim. Cosmochim. Acta 2003, 67, 2977-2992.

9. Fredrickson, J. K.; Zachara, J. M.; Kukkadapu, R. K.; Gorby, Y. A.; Smith, S. C.; Brown, C. F. Biotransformation of NiSubstituted Hydrous Ferric Oxide by an Fe(III)-Reducing Bacterium. Environ. Sci. Technol. 2001, 35, 703-712.

10. Moon, J.-W.; Yeary, L. W.; Rondinone, A. J.; Rawn, C. J.; Kirkham, M. J.; Roh, Y.; Love, L. J.; Phelps, T. J. Magnetic Response of Microbially Synthesized Transition Metal- and Lanthanide-Substituted Nanosized Magnetites. J. Magn. Magn. Mater. 2007, 313, 283-292.

11. Matsunaga, T.; Okamura, Y.; Tanaka, T. Biotechnological Applications of Nano-Scale Engineered Bacterial Magnetic Particles. J. Mater. Chem. 2004, 14, 2099-2105.

12. Lang, C.; Schüler, D. Biogenic Nanoparticles: Production, Characterization, and Application of Bacterial Magnetosomes. J. Phys.: Condens. Mater. 2006, 18, S2815S2828.

13. Matsunaga, T.; Suzuki, Y.; Tanaka, M.; Arakaki, A. Molecular Analysis of Magnetotactic Bacteria and Development of Functional Bacterial Magnetic Particles for NanoBiotechnology. Trends Biotechnol. 2007, 25, 182-188.

14. Prozorov, T.; Palo, P.; Wang, L.; Nilsen-Hamilton, M.; Jones, D.; Orr, D.; Mallapragada, S. K.; Narasimhan, B.; Canfield, P. C.; Prozorov, R. Cobalt Ferrite Nanocrystals: OutPerforming Magnetotactic Bacteria. ACS Nano 2007, 1, 228-233.

15. Staniland, S.; Williams, W.; Telling, N. D.; van der Laan, G.; Harrison, A.; Ward, B. Controlled Cobalt Doping of Magnetosomes In Vivo. Nat. Nanotechnol. 2008, 3, 158-162.

16. Roh, Y.; Vali, H.; Phelps, T. J.; Moon, J.-W. Extracellular Synthesis of Magnetite and Metal-Substituted Magnetite Nanoparticles. J. Nanosci. Nanotechnol. 2006, 6, 3517-3520.

17. Scherrer, P. Estimation of Size and Internal Structure of Colloidal Particles by Means of Rontgen Rays. Nachr. Ges. Wiss. Goettingen 1918, 96-100.

18. van der Laan, G.; Kirkman, I. W. The $2 p$ Absorption Spectra of $3 d$ Transition Metal Compounds in Tetrahedral and Octahedral Symmetry. J. Phys.: Condens. Mater. 1992, 4, 4189-4204. 
19. van der Laan, G.; Thole, B. T. Strong Magnetic X-ray Dichroism in $2 p$ Absorption Spectra of $3 d$ Transition Metal Ions. Phys. Rev. B 1991, 43, 13401-13411.

20. Coker, V. S.; Pearce, C. I.; Lang, C.; van der Laan, G.; Pattrick, R. A. D.; Telling, N. D.; Schuler, D.; Arenholz, E.; Lloyd, J. R. Cation Site Occupancy of Biogenic Magnetite Compared to Polygenic Ferrite Spinels Determined by X-ray Magnetic Circular Dichroism. Eur. J. Mineral. 2007, 19, 707-716.

21. Carvallo, C.; Sainctavit, P.; Arrio, M.-A.; Menguy, N.; Wang, Y.; Ona-Nguema, G.; Brice-Profeta, S. Biogenic vs. Abiogenic Magnetite Nanoparticles: A XMCD Study. Am. Mineral. 2008, 93, 880-885.

22. van der Laan, G.; Arenholz, E.; Chopdekar, R. V.; Suzuki, Y. Influence of Crystal Field on Anisotropic X-ray Magnetic Linear Dichroism at the $\mathrm{Co}^{2+} L_{2,3}$ Edges. Phys. Rev. B 2008, 77, 064407-1/11.

23. Zhang, X. X.; Hernandez, J. M.; Tejada, J.; Ziolo, R. F. Magnetic Properties, Relaxation, and Quantum Tunneling in $\mathrm{CoFe}_{2} \mathrm{O}_{4}$ Nanoparticles Embedded in Potassium Silicate. Phys. Rev. B 1996, 54, 4101-4106.

24. Verwey, E. J. W. Electronic Conduction in Magnetite $\left(\mathrm{Fe}_{3} \mathrm{O}_{4}\right)$ and its Transition Point at Low Temperatures. Nature 1939, 144, 327-328.

25. Goya, G. F.; Berquo, T. S.; Fonseca, F. C.; Morales, M. P. Static and Dynamic Magnetic Properties of Spherical Magnetite Nanoparticles. J. Appl. Phys. 2003, 94, 3520-3528.

26. Brabers, V. A. M.; Walz, F.; Kronmüller, H. Impurity Effects Upon the Verwey Transition in Magnetite. Phys. Rev. B 1998, 58, 14163-14166.

27. Bean, C. P. Hysteresis Loops of Mixtures of Ferromagnetic Micropowders. J. Appl. Phys. 1955, 26, 1381-1383.

28. Granqvist, C. G.; Buhrman, R. A. Ultrafine Metal Particles. J. Appl. Phys. 1976, 47, 2200-2219.

29. von Helmolt, R.; Wecker, J.; Samwer, K. Calculation of Particle Size from Magnetization and Resistance Curves in Giant Magnetoresistive Heterogeneous Alloys. Phys. Status Solidi B 1994, 182, K25.

30. Fortin, J.-P.; Wilhelm, C.; Servais, J.; Menager, C.; Bacri, J.-C.; Gazeau, F. Size-Sorted Anionic Iron Oxide Nanomagnets as Colloidal Mediators for Magnetic Hyperthermia. J. Am. Chem. Soc. 2007, 129, 2628-2635.

31. Chikazumi, S. The Physics of Ferromagnetism, 2nd ed.; Oxford University Press: Oxford, 1997.

32. Ibusuki, T.; Kojima, S.; Kitakami, O.; Shimada, Y. Magnetic Anisotropy and Behaviours of Fe Nanoparticles. IEEE Trans. Magn. 2001, 37, 2223-2225.

33. Lopez, J. L.; Pfannes, H.-D.; Paniago, R.; Sinnecker, J. P.; Novak, M. A. Investigation of the Static and Dynamic Magnetic Properties of $\mathrm{CoFe}_{2} \mathrm{O}_{4}$ Nanoparticles. J. Magn. Magn. Mater. 2008, 320, e327-e330.

34. Rosensweig, R. E. Heating Magnetic Fluid with Alternating Magnetic Field. J. Magn. Magn. Mater. 2002, 252, 370-374.

35. Craik, D. J. Intrinsic and Technical Magnetic Properties of Oxides. In Magnetic Oxides; Craik, D. J., Ed.; Wiley: London, 1975; pp 1-96.

36. Coey, J. M. D. Noncollinear Spin Arrangement in Ultrafine Ferromagnetic Crystallites. Phys. Rev. Lett. 1971, 27, 1140-1142.

37. Lovley, D. R.; Phillips, E. J. P. Availability of Ferric Iron for Microbial Reduction in Bottom Sediments of the Freshwater Tidal Potomac River. Appl. Environ. Microbiol. 1986, 52, 751-757.

38. Lloyd, J. R.; Leang, C.; Hodges Myerson, A. L.; Coppi, M. V.; Cuifo, S.; Methe, B.; Sandler, S. J.; Lovley, D. R. Biochemical and Genetic Characterization of PpcA, a Periplasmic c-type Cytochrome in Geobacter sulfurreducens. Biochem. J. 2003, 369, 153-161.

39. Arenholz, E.; Prestemon, S. O. Design and Performance of an Eight-Pole Resistive Magnet for Soft X-ray Magnetic Dichroism Measurements. Rev. Sci. Instrum. 2005, 76, 083908/1-8

40. Pattrick, R. A. D.; van der Laan, G.; Henderson, C. M. B.; Kuiper, P.; Dudzik, E.; Vaughan, D. J. Cation Site Occupancy in Spinel Ferrites Studied by X-ray Magnetic Circular
Dichroism: Developing a Method for Mineralogists. Eur. J. Mineral. 2002, 14, 1095-1102. 